\title{
Unleashing the Spatial Domain in Optical Fiber Communications
}

\author{
D.J. Richardson \\ Optoelectronics Research Centre, University of Southampton, Highfield, Southampton, SO17 1BJ, UK. \\ djr@orc.soton.ac.uk
}

\begin{abstract}
SDM offers the potential for ultrahigh information-flux optical communications at petabit/s level capacities, and also reduced costs per-bit. I review progress to date and discuss some of the technological/commercial challenges and opportunities that lie ahead.
\end{abstract}

\section{INTRODUCTION}

Concerns of a future capacity crunch due to the ever increasing data traffic on the world's communications networks has ignited interest in developing new forms of optical fiber transmission technology capable of much higher per-fiber transmission capacities and lower costper-bit than current single-mode fiber (SMF) technology. Given that all other signaling dimensions i.e. wavelength, polarization, phase and amplitude are already close to maximally exploited in state-of-the-art SMF based systems, the primary focus of recent research has been on better exploiting the spatial domain: more specifically on developing fibers that support multiple distinguishable spatial information channels - a generic approach referred to as Space Division Multiplexing (SDM) [1]. The fundamental attractions of SDM are significant. Firstly, it offers a means to increase the information flow-per-unitarea (i.e. information flux) by allowing a more intimate proximity of information channels than conventional fibers (or indeed bundles of conventional fibers), and thereby increased per-fiber capacity. Secondly, the increased information flux should allow for far higher levels of device integration (e.g. amplifiers, transmitters and receivers) than possible in SMF based systems, thereby promising a lower cost-per-bit and reduced energy demands. The technical challenges in realizing these promises though are profound.

\section{Technical Progress towards Higher PeR- FIBER CAPACITIES}

Conceptually at least, the most straightforward approach to SDM is to incorporate multiple independent singlemode cores within the fiber cross-section and this has received a large amount of interest over the past three years, particularly in Japan. Highly impressive advances have been made: both in terms of the underpinning technology (transmission fibers, amplifiers and supporting components), and the associated transmission experiments themselves. The progress is perhaps best appreciated by reference to Fig.1, where the highest capacity and highest capacity $\mathrm{x}$ length product results reported at the postdeadline session of the annual conference on Optical Fiber Communications (OFC) for the past 30 years are plotted (black), along with the latest SDM-based results (red) (all of which have been obtained using MCF). It can be seen that MCF technology has already enabled a 10fold increase in record capacity (now up to $1 \mathrm{Pbit} / \mathrm{s}$ in a 12-core fiber [2]), and equivalent performance in terms of capacity x length product [3] relative to SMF. Mechanical fiber reliability issues are likely to limit the total number of independent cores (and hence overall capacity scaling factor) to $<\sim 20$, and the migration to higher core counts will require mitigation/accommodation of inter-core cross-talk. Work on this later topic is underway, but so far only 3-core, high-core-density fibers operating in the strong coupling limit have been studied (referred to as coupled-core fibers)[4]. Operation of these fibers has much in common with the mode division multiplexing (MDM) approach discussed below.

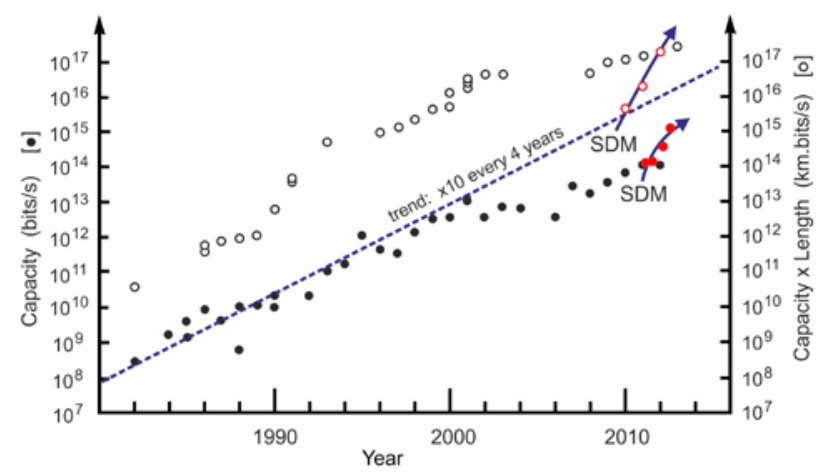

Fig.1. the highest capacity and highest capacity $\mathrm{x}$ length product results from the postdeadline sessions of the Optical Fiber Communications (OFC) conference over the past 30 years. (SDM results shown in red, SMF results in black). A detailed table of the most significant SDM transmission results to date is provided in [1].

Progress in the alternative approach of using MDM (in which the individual modes of a few-mode fiber (FMF) are used to define the information channels) has also been hugely impressive. Coupling of signal power between the different physical modes used within the fiber (in conjunction with the fiber's Differential Mode Group Delay) is a critical issue, and Multiple-Input, MultipleOutput (MIMO) digital signal processing (DSP) techniques are generally required to recover the data [5]. Net-capacities in excess of $50 \mathrm{Tbit} / \mathrm{s}$ have already been 
achieved (for a total of 3 modes in an amplified 115km long link) [6], and the transmission of up to 6 modes has been demonstrated over $177 \mathrm{~km}$ [7]. Amplified MDM transmission in FMF over distances in excess of $700 \mathrm{~km}$ has also now been reported [8].

In order to gain more significant capacity increases it is possible to overlay MDM directly on to the MCF approach. In a first proof-of-principle implementation a fiber capable of supporting up to 21 different spatial modes (7 cores each, supporting 3 modes) was demonstrated [9]. Soon after, a hybrid MCF comprising 12 single-mode cores and two few-mode cores supporting three spatial modes was demonstrated, allowing transmission at $1.05 \mathrm{Pbit} / \mathrm{s}$ (albeit over just 3km) [10]. Nevertheless this experiment was the first to achieve a spectral efficiency beyond $100 \mathrm{bit} / \mathrm{s} / \mathrm{Hz}$.

\section{Progress Towards Cost Reduction}

Work towards proving the potential cost reduction benefits of SDM is also underway, however it is understandably less advanced than the research demonstrating capacity enhancement. Arguably, the greatest progress to date has been made in the area of amplification, with both MCF and FMF optical amplifiers demonstrated and shown to be capable of supporting long haul transmission $[2,8]$. However, an SDM EDFA with the full functionality associated with a conventional SMF EDFA, whilst simultaneously allowing a reduced component count, has yet to be demonstrated. Cladding pumping appears particularly attractive for SDM amplification since it naturally allows the sharing of pump radiation across multiple amplifying data-pathways, however achieving the required levels of control and performance (e.g. low noise figure and a flat gain spectrum across the full C-band) appears to be challenging. Work on integrated signal Mux/Demux devices, transmitters and receivers, is also progressing well and promises cost-per-bit reduction opportunities. However, this integration work is still in its infancy and requires substantial further development activity.

\section{OUTSTANDING TECHNICAL CHALLENGES AND THE ROUTE TO ADOPTION}

Whilst much technical progress has undeniably been made, many fundamental issues associated with SDM in its various forms remain to be understood, and numerous technological and economic challenges will then need to be overcome if SDM is ever to be adopted commercially. For example, from a scientific perspective: nonlinear effects in both FMFs and MCFs need to be better understood and the system implications quantified; approaches to reduce the MIMO DSP processing complexity to levels compatible with real-time signal processing need to be developed; and a whole slew of issues remain to be resolved in order to realize practical SDM components and subsystems. In addition, a detailed understanding of SDM networking and the associated component and control strategies required has yet to be developed, and work is only just beginning on these topics. From an economic perspective it will be necessary to prove that volume manufacture of high-performance
SDM fibers and components is possible and that these can provide a significant cost-per-bit benefit for system operators. It goes without saying that the performance and reliability/robustness of SDM systems will need to be proven to be as good, if not better, than current SMF based systems. System operators will also need to develop a graceful upgrade strategy if they are ever to start installing SDM technology. One possible scenario is depicted in Fig.2.

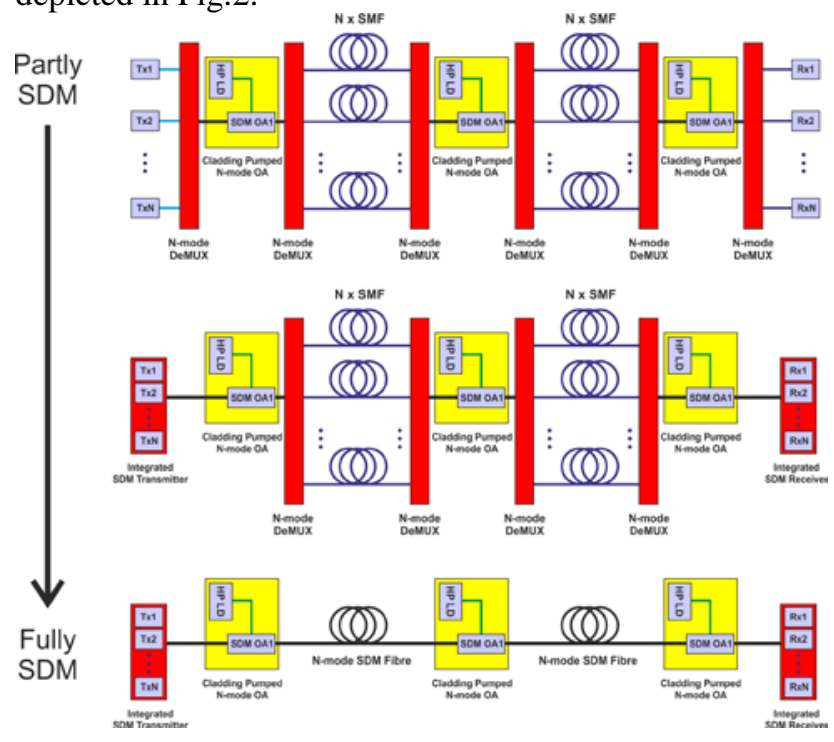

Fig.2. A possible adoption scenario for SDM technology. System providers may gradually introduce SDM components (e.g. SDM amplifiers, transmitters and receivers) into SMF systems in order to benefit from the short term cost savings possible, before upgrading to fully SDM systems once new fiber deployments become unavoidable.

\section{CONCLUSIONS}

Rapid progress has been made in SDM transmission over the past 3 years, however much further work needs to be undertaken if per-channel reliability and performance competitive with existing SMF links, and a lower costper-bit are to be realized. Moreover, major manufacturing and economic challenges will also need to be overcome if SDM technology is ever to be deployed commercially. However, irrespective of the ultimate outcome, the next few years promise to be a busy and exciting time in optical fiber research. It is also important to note the new technology and capabilities under development are already opening up new opportunities in both related (e.g. datacomms), and adjacent fields (e.g. high power fiber lasers and optical sensing). Some of these opportunities will be described during the presentation.

\section{REFERENCES}

[1] D.J. Richardson et al., Nature Photonics 2013 (http://arxiv.org/abs/1303.3908)

[2] Takara, H. et al. Proc. ECOC 2012, Th3.C.1.

[3] Takahashi, H. et al., Proc. ECOC 2012, Th.3.C.3.

[4] Ryf, R. et al. IEEE PTL 23, 1469-1471, (2011).

[5] Randel, S. et al. Opt. Exp. 19, 16697-16707, (2011).

[6] Sleiffer, V.A.J.M. et al. Proc. ECOC 2012, Th.3.C.4.

[7] Ryf, R. et al. Proc. OFC-NFOEC 2013, PDP5A.1.

[8] Ip E. et al. Proc. OFC-NFOEC 2013, PDP5A.2.

[9] Xia, C. et al. IEEE PTL, 24, 1914-1916, (2012).

[10] Qian, D. et al. Proc. FiO 2012, FW6C.3. 
